\title{
Spermatogénèse et Paraplégie
}

Albert LERICHE, Marie-Françoise MONNET, Bertrand POGU, Geneviève PILONCHERY

Hospices Civils de Lyon; Hôpital Henry Gabrielle, Unité d'Urologie, Route de Vourles, 69230 Saint Genis Laval

\section{RESUME}

Dans cet article, vont être passées en revue les possibilités de procréation du paraplégique.

Un premier chapitre traitera du fonctionnement testiculaire avec l'étude des dosages hormonaux et de l'histologie testiculaire (étude portant sur 100 patients). Au vue de la biopsie testiculaire, il y a une lésion exocrine, d'origine neurologique, parfois irréversible. Par contre, les lésions du testicule endocrine ont été réversibles sous stimulation ou spontanément.

Un deuxième chapitre traitera des différentes techniques pour obtenir une éjaculation et l'analyse du spermogramme. Les techniques utilisées étant : le vibro-massage, l'électro-éjaculation, l'éjaculation par stimulation pharmacologique. L'étude porte sur 124 tests éjaculatoires, pour lesquels ont été obtenues 64 éjaculations antégrades $(52 \%)$ et 4 éjaculations rétrogrades (3\%). Le spermogramme obtenu a montré une numération des spermatozoïdes supérieure à 20 millions par $\mathrm{ml}$ dans 35 cas (soit $51,5 \%$ ) et inférieure à 5 millions dans 11 cas (soit 16\%).

Mots Clés : Paraplégie, procréation, éjaculation

\section{INTRODUCTION}

Les possibilités de procréation restent une des préoccupations essentielles et rapides du jeune paraplégique, bien avant de savoir s'il pourra récupérer une éjaculation. Les médias ont largement diffusé les informations sur les techniques de traitement de l'impuissance, d'insémination artificielle et même de ponction épididymaire pour que le problème de l'éjaculation puisse paraitre secondaire au paraplégique, s'il sait qu'il pourra satisfaire sexuellement sa partenaire et la féconder quel que soit le moyen utilisé.

Le jeune paraplégique veut donc d'emblée savoir quel est l'état de sa spermatogenèse, dès son hospitalisation immédiate, avant sa sortie, dès qu'il a acquis la conviction qu'il restera paraplégique.

Il y a quelques années, nous avons commencé par essayer de récupérer du sperme pendant cette période initiale post-traumatique, mais sans succès.

Nous avons donc essayé de faire le bilan de l'état testiculaire sans éjaculation en associant bilan hormonal et biopsie testiculaire chez 100 patients.

\section{LE FONCTIONNEMENT TESTICULAIRE}

\section{Matériel}

Notre matériel porte sur 100 blessés médullaires répartis selon le niveau neurologique en :

- 27 tétraplégiques ;

- 23 paraplégiques dorsaux hauts ;

- 10 paraplégiques en D8 D9 ; 
- 6 paraplégiques en D10 D11;

- 18 paraplégiques en $\mathrm{D} 12 \mathrm{~L} 1$;

- 16 syndromes de la queue de cheval ou de l'épicone.

Nous avons partagé le groupe des paraplégiques dorsaux en quatre, en effet à chacun de ces niveaux se posent des problèmes de miction, d'érection et d'éjaculation différents.

La lésion neurologique était complète dans $58 \%$ des cas et incomplète dans $42 \%$.

La neuro-vessie est de type :

- centrale dans $58 \%$ des cas ;

- périphérique dans $20 \%$ des cas ;

- mixte dans $4 \%$ des cas ;

- normale dans 7\% des cas ;

- une seule vessie n'a pas été expertisée soit $1 \%$.

Les érections sont présentes dans $70 \%$ des cas, absentes dans $25 \%$ et inconnues dans $5 \%$ des cas.

La libido est normale chez 64 patients, absente 14 fois et inconnue 22 fois.

La vie sexuelle est normale dans $27 \%$ des cas, absente dans $51 \%$ et inconnue dans $22 \%$ des cas.

L'âge des patients se répartit ainsi :

- 16 ayant moins de 20 ans ;

- 42 ayant entre 20 et 29 ans ;

- 19 ayant entre 30 et 39 ans ;

- 11 ayant entre 40 et 49 ans ;

- 5 ayant entre 50 et 59 ans ;

- 7 ayant plus de 60 ans.

L'âge de la lésion médullaire :

- 48 malades ayant moins d'un an de paraplégie ;

- 23 ayant un an ;

- 11 ayant deux ans ;

- 5 ayant trois ans ;

- 13 ayant plus de quatre ans.

Chez ces malades, les bilans les plus précis

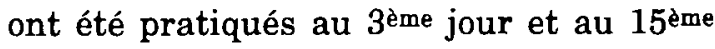

jour de la paraplégie et le bilan le plus tardif au bout de 22 ans de paraplégie.

\section{Méthodes}

Le bilan biologique comporte pour tous les patients des dosages urinaires des 17 cétostéroïdes et de la testostérone sur des urines de 24 heures et recueillis sur du cyanure de mercure. Les prélèvements se font les $1^{\mathrm{er}}, 4^{\text {ème }}$ et $7^{\mathrm{èm} \theta}$ jours de la semaine. Les trois premiers jours, la sécrétion surrénalienne est freinée par 8 comprimés de Dectancyl 0,5 mg (Dexaméthazone) dans la journée. Les trois jours suivants en continuant la freination surénalienne on réalise une stimulation testiculaire. Pour cela on pratique une injection intramusculaire de Gonadotrophine Chorionique ISH 5000 U.I., par jour et ceci dans les zones sensibles afin d'éviter les risques d'escarres.

Les dosages plasmatiques radio-immunologiques de la testostérone, de FSH et de $\mathrm{LH}$, de la prolactine et de l'œstradiol et le dosage des hormones hypophysaires après stimulation par le releasing-factor : LH.RH ont été pratiqués.

Les hormones hypophysaires FSH et LH sont marquées à l'iode 125 comme la prolactine.

Le dosage des stéroides plasmatiques est fait par extraction à l'éther sans chromatographie.

La biopsie testiculaire a été pratiquée par une petite incision scrotale. On a noté un hématome sur 63 biopsies.

\section{Résultats}

\section{a) Le bilan biologique}

Les dosages statiques :

- Les 17 Ceto urinaires sont :

- normaux : compris entre 12 et $18 \mathrm{mg} / 24$ h dans $49 \%$;

- bas : 20\% avec un taux minimum de 3 $\mathrm{mg} / 24 \mathrm{~h}$;

- hauts : $31 \%$ avec un taux maximum de $30 \mathrm{mg} / 24 \mathrm{~h}$. 
- La testostérone urinaire est :

- normale : entre 40 et $100 \mathrm{mg} / 24 \mathrm{~h}$ dans $45 \%$ des cas,

- basse : $44 \%$ avec un taux minimum de $10 \mathrm{mg} / 24 \mathrm{~h}$,

- haute : $11 \%$ avec un taux maximum de $125 \mathrm{mg} / 24 \mathrm{~h}$.

- La testostérone sanguine est à un taux :

- normal : entre 3,5 et $8,5 \mathrm{mg} / \mathrm{ml}$ dans $76 \%$ des cas,

- bas : $20 \%$ avec un minimum de $1 \mathrm{mg} / \mathrm{ml}$, - élevé : $4 \%$ avec un maximum de 10 $\mathrm{mg} / \mathrm{ml}$.

- Les taux de FSH sont :

- normaux : compris entre 2,5 et 13 MUI/ml dans $62 \%$ des cas,

- bas : $17 \%$ avec un minimum nul,

- hauts : $21 \%$ avec un maximum de 35 $\mathrm{MUI} / \mathrm{ml}$.

- Les taux de LH sont :

- normaux : compris entre 4 et $20 \mathrm{MUL} / \mathrm{ml}$ dans $75 \%$ des cas,

- bas : $8 \%$ avec un minimum nul,

- hauts : $17 \%$ avec un maximum de 35 $\mathrm{MUI} / \mathrm{ml}$.

- La prolactine plasmatique est à un taux :

- normal : entre 10 et $30 \mathrm{mg} / \mathrm{ml}$ dans $60 \%$ des cas,

- bas : $30 \%$ avec un minimum nul,

- haut : $10 \%$ avec un maximum de 60 $\mathrm{mg} / \mathrm{ml}$.

- Le dosage de l'œstradiol plasmatique est à un taux:

- normal : compris entre 20 et $45 \mathrm{pg} / \mathrm{ml}=$ $65 \%$,

- bas : dans 10\% avec un minimum de 4 $\mathrm{pg} / \mathrm{ml}$,

- haut : 25\% avec un maximum de 120 $\mathrm{pg} / \mathrm{ml}$.

Les dosages dynamiques :

- Urinaires :

L'épreuve de freination : la stimulation est positive dans $71 \%$ des cas pour la sécrétion des 17 Cétostéroïdes urinaires et dans $95 \%$ des cas pour la testostérone. Dans $65 \%$ des cas le taux de testostérone est multiplié par 3 et la réponse à la stimulation est alors qualifiée d'explosive.

\section{- Plasmatiques :}

Le test au LHRH permet de classer les réponses en plusieurs types :

. normal : taux de base de FSH et LH normaux ; après injection de $100 \mathrm{mgIV}$ de LHRH, le taux de LH présente un pic de $47,3 \pm 6,5 \mathrm{MUL} / \mathrm{ml}$ à la 30 ème minute et le taux de FSH un pic retardé à la 60 ème minute de 17,2 $\pm 7,5 \mathrm{MUL} / \mathrm{ml}$,

. périphérique : avec taux de base de FSH et LH élevé et réponse importante à la stimulation,

. hypophysaire : taux bas ; réponse faible ou normale,

. hypothalamique : taux bas, réponse élevée et retardée.

Les types de réponses au LHRH se répartissent ainsi : normale

périphérique

hypophysaire

hypothalamique

\section{FSH LH}

$41 \% \quad 38 \%$

$18 \% \quad 18 \%$

$38 \% \quad 9 \%$

$3 \% \quad 35 \%$
Le test au TRH permet d'obtenir un taux de prolactine supérieur à $30 \mathrm{mg}$ à la 15 ème minute après l'injection dans $50 \%$ des cas.

L'hyperprolactinémie réactionnelle est considérée comme pathologique quand elle est supérieure à $100 \mathrm{pg} / \mathrm{ml}$ soit dans $15 \%$ des cas.

Le test aux gonadotrophines est toujours positif pour l'œstradiol dont le taux s'élève toujours.

\section{b) L'histoire testiculaire}

- Spermatogenèse :

- Active : $51 \%$

- Ralentie : $35 \%$

- Bloquée : $21 \%$ 
- Associée à des tubes atrophiques : 23,5\%

- Absente : $28 \%$

- Encombrement des tubes par des éléments immatures : $35 \%$

- Membrane basale :

- Normale : $17,5 \%$

- Epaissie et scléreuse : 82,5\%

- Cellules de Sertoli :

- Rares : $2 \%$

- Abondantes et hyperplasiques : $66 \%$

- Vacuolisées :40\%

- Tissu interstitiel :

- Scléreux : $63 \%$

- Normal : $25 \%$

- CEdémateux : 8\%

- Scléro-œdémateux : $4 \%$

- Cellules de Leydig :

- Normales : $51 \%$

- Absences : $6 \%$

- Rares : $18 \%$

- Hyperplasiques : $10 \%$

- Peu fonctionnelles : $10 \%$

\section{Discussion}

a) L'atteinte de la lignée germinale testiculaire après section médullaire avait déjà été démontrée tant expérimentalement que cliniquement (HoRNE [15], KLEIN, BORS, DALAGE, LERICHE). Les lésions en dessous de L2 avaient déjà la réputation de respecter la spermatogenèse (BoRS [4], LERICHE [17], DALAGE [11]). La conservation de la spermatogenèse pour les lésions D10-D11 est un élément nouveau, surprenant, puisqu'il s'agit des métamères assurant l'innervation sensitive du testicule.Un travail parallèle (BÉRARD) [2] sur la sensibilité testiculaire après paraplégie n'a pas permis d'établir un pronostic sur la spermatogenèse contrairement à ce qu'affirmait TsUJI [25]. Le pronostic fonctionnel testiculaire ne semble pas meilleur lorsque la sensibilité testiculaire est conservée. L'apparition très précoce des lésions testiculaires avait déjà été décrite (4ème jour : KLEIN) [17]. Le nombre de biopsies itératives à long terme est insuffisant pour juger de l'évolution d'une récupération.
Certains spermogrammes paraissent acceptables avec des histologies très pathologiques. La desquamation de l'épithélium germinatif fait penser à une nécrose d'origine vasomotrice. Ce phénomène est comparable à celui obtenu après vasectomie. On pourrait donc espérer une récupération de cette spermatogenèse. Il faut remarquer la présence de spermatogenèse active dans des paraplégies anciennes et chez des paraplégiques âgés. Le temps n'est donc pas le facteur déterminant de la lésion testiculaire.

Le taux de FSH ne suffit pas à apprécier la qualité de la spermatogenèse mais les taux élevés sont de mauvais pronostic.

b) L'altération du tissu testiculaire endocrine après paraplégie n'est pas classique. Elle apparaît ici très nettement avec un œdème puis une fibrose du tissu interstitiel pouvant coïncider avec une conservation de la spermatogenèse et des sécrétions hormonales anormales avec un dysfonctionnement de l'axe hypothalamo-hypophysaire. La biopsie n'a évidemment aucune valeur pour apprécier la fonction des cellules de Leydig puisqu'elle est ponctuelle, en lieu et en temps. La sécrétion de testostérone peut être normale avec des cellules de Leydig rares. La sensibilité de ce tissu endocrine aux hormones hypophysaires doit être discutée. Le taux de testostérone coïncidant avec des taux élevés de LH ne devraient pas augmenter à l'administration de gonadotrophines exogènes. Il faut donc penser, dans ces cas là, que le tissu testiculaire est insensible aux hormones endogènes. Peut-être s'agit-il d'un problème vasculaire : les hormones hypophysaires sont encore à un taux insuffisant du fait d'une mauvaise pénétration vasculaire alors que les hormones exogènes sont à des taux 100 à 1000 fois supérieurs et ont donc plus de chances de pénétrer.

L'existence de la sclérose du tissu interstitiel et des vaisseaux et l'élévation du taux de testostérone lors de la stimulation de la sécrétion de FSH LH par le LHRH sont en faveur de cette hypothèse. 
En fait on sait maintenant que la biopsie testiculaire ne reflète qu'un moment donné et que des atrophies testiculaires complètes après chimiothérapie ont pu récupérer. Il est donc vraisemblable que c'est la même chose avec la paraplégie.

COMARr et Bors [10] ayant éliminé l'influence des infections génito-urinaires, de la lithiase, des escarres et des infections diverses sur la lignée germinale, nous n'avons pas cru bon de reprendre cette enquête. Nous n'avons pas non plus recherché l'effet de la Furadoïne du fait de la précocité des lésions, mais peut-être a-t-elle un effet sur l'absence de récupération ? MORALES [18] avait démontré qu'il n'existait pas d'hyperthermie du scrotum du paraplégique. Nous n'avons donc pas pratiqué de thermographie. Les doses d'exposition aux rayons $\mathrm{X}$ de nos malades sont très inférieures aux doses toxiques mais peut-être ont-elles une influence sur la sclérose ou sur la récupération? Les anticorps anti-testiculaires ou anti-spermatozoïdes n'ont pas été recherchés alors qu'il existe une extravasation et un passage sanguin possible lors du traumatisme médullaire. L'utilisation d'un anti-prolactinémique (CB 159) dans les cas d'hyperprolactémie réactionnelle n'a pas permis la récupération d'une spermatogenèse. On note $12 \%$ d'hyper-prolactinémie contre $10 \%$ dans la population masculine stérile non paraplégique.

\section{L'ANALYSE DU SPERMOGRAMME}

Les moyens d'obtenir une éjaculation seront analysés par le Dr EGON. Nous allons simplement essayer d'analyser les résultats de 124 tests éjaculatoires pour lesquels nous avons obtenu 64 éjaculations antégrades $(52 \%)$ et 4 éjaculations rétrogrades (3\%).

Le spermogramme ainsi obtenu a montré une numération des spermatozoïdes supérieure à 20 millions par ml dans 35 cas (soit 51,5\%) et inférieure à 5 millions dans 11 cas (soit 16\%).

Il faut rappeler que l'OMS considère l'oligospermie à partir de 20 millions, mais que les chances de fécondation sont quasiment identiques jusqu'à 5 millions $/ \mathrm{ml}$.

Existe-t-il réellement une différence de qualité du sperme selon le mode d'obtention de l'éjaculation?

\section{Le vibro-massage :}

Les résultats sont assez comparables à ceux de la littérature puisque BECKERMAN [1] à partir d'une analyse de 10 articles sélectionnés relève $60 \%$ d'éjaculations obtenues par vibro-massage (257/428) dont $88,3 \%$ antégrades, sans anomalie de volume et de qualité du sperme.

Brindley G.S. [5], Beretta G. [3], Siosteen A. [22] notent une amélioration avec la répétition des stimulations de la qualité du sperme, en particulier de la numération, de la mobilité et de la forme normale, mais aussi du taux de fructose et de phosphatases acides.

Au contraire, ENGH E. [12] ne note aucune amélioration et, personnellement, nous avons vu au contraire des détériorations de la qualité du sperme avec la répétition du vibro-massage dans 2 cas.

\section{L'électro-éjaculation :}

La première grossesse a été rapportée par THOMAS [24] en 1975 et la première naissance d'un enfant vivant en 1978 par FraNçOIS [13].

L'analyse des résultats est souvent difficile du fait des différentes techniques d'électroéjaculation. Dans la littérature, une éjaculation antégrade est obtenue, en général, dans 36 à $75 \%$ des cas.

Sur 154 patients, BRINDLEY rapporte 7 émissions de sperme par le méat (45\%), 27 éjaculations rétrogrades (18\%), soit 97 résultats positifs $(63 \%), 42$ échecs $(27 \%)$ et 15 douleurs ne permettant pas l'usage de l'électro-éjaculateur. SARKARATI [6] utilisant le même matériel que BRINDLEY [21] relève seulement 8 éjaculations antégrades sur 22 cas (36\%). HALSTEADT note 9 (75\%) éjaculations antégrades sur 12 cas, et WARNER 14 
(64\%) sur 22 cas ce qui est proche des $61 \%$ d'éjaculations antégrades (11/18 tests) de notre série. Pour sa part, OHL [19] a 71\% de résultats positifs définis comme une éjaculation antégrade avec plus de 10 millions de spermatozoïdes dont plus de 1 million de mobiles. Bennet expose ses résultats en fonction du niveau neurologique : $54 \%$ de résultats positifs lors de lésion cervicale $(7 / 13), 21 \%$ thoracique (14) et $33 \%(1 / 3)$ au niveau sacré. Au contraire, OHL trouve que l'éjaculation est plus fréquente lors de lésion thoracique. De la même façon, nous avons obtenu une éjaculation antégrade dans $70 \%$ (7/10 tests) des lésions de niveau T1-T10.

Contrairement au vibro-massage, l'efficacité de l'électro-éjaculation est identique avant ou après un délai de 6 mois par rapport à la lésion. Dans notre travail, des éjaculations antégrades ont été obtenues dans 100\% (4/4 tests) des tests pratiqués avant 6 mois et $50 \%$ (7/14 tests) des tests pratiqués après 6 mois.

La qualité du sperme, notamment la mobilité des spermatozoïdes, est moins bonne après électro-éjaculation. Selon BRINDLEY, les deux explications aux meilleurs résultats du vibro-massage sont l'absence de contamination urinaire et la rareté des éjaculations partiellement rétrogrades. Cependant, LISENMEYER a montré que le courant pouvait avoir un effet adverse sur la mobilité des spermatozoïdes. Ainsi, il existe en laboratoire une diminution de la mobilité pour tous les spermes exposés à un courant de $100 \mathrm{~mA}$ pendant deux minutes par rapport à ceux qui n'y sont pas exposés. Nous avons obtenu $36 \%$ d'éjaculations antégrades et rétrogrades avec plus de 20 millions de spermatozoïdes, dont plus de $20 \%$ mobiles à la suite d'une électro-éjaculation. Certains auteurs relèvent que la qualité du sperme, essentiellement la numération et le pourcentages de formes anormales, tend à s'améliorer avec la répétition des essais. Ce dernier point n'est pas confirmé par SARKARATI. Par ailleurs, il n'existe pas de corréla- tion entre cette qualité et le délai depuis la lésion.

La stimulation directe hypogastrique de Brindley, à propos de 7 observations, a permis d'obtenir 5 grossesses par insémination du sperme ainsi obtenu, dont 2 ont été menées à terme chez les femmes de 4 patients.

\section{L'éjaculation par stimulation phar- macologique :}

a) La Néostigmine (Prostigmine) : c'est en 1947 que Guttman rapportait la possibilité d'induire une éjaculation chez le blessé médullaire grâce à l'injection intra-thécale de Néostigmine. En 1971, GutTman et WALSH notaient $59,7 \%$ de succès chez 70 blessés médullaires avec lésions suprasacrées. La majorité des mauvais résultats était enregistrée pour des lésions comprises entre T10 et L4. Des grossesses ont été ainsi obtenues par SPIRA [23], CHAPELLE [9] et OTANi [20].

b) La Physostigmine (Eserine) : en 1988, CHAPELLE [8] relève 75 résultats positifs sur 135 tests $(55,5 \%)$ à la Physostigmine sous cutanée chez des patients porteurs de lésions médullaires complètes. Seuls 3 patients porteurs de lésions médullaires complètes des métamères T12-L2 ont eu des résultats positifs. Avec cette technique, récemment BAKKER notait 5 bons résultats sur 10 tests $(50 \%)$. Nos résultats sont proches de ces derniers que ce soit en usage intra-veineux $(45,5 \%)$ ou sous-cutané (46\%).

Pour la Physostigmine sous cutanée, les éjaculations antégrades ont été obtenues dans 4 cas sur 5 au niveau cervical (80\%), 5 sur 10 pour des lésions de T1 à T10 (50\%) et aucune fois sur 9 tests pour des niveaux de T11 à L1. Ces bons résultats au niveau cervical se retrouvent aussi pour la Physostigmine intra-veineuse (4 éjaculations antégrades sur 6 tests).

Le spermogramme est de bonne qualité une fois sur deux. 
Tableau 1 : Numération des spermatozoïdes suivant les méthodes utilisées.

\begin{tabular}{lccc}
\hline Méthodes & Nb de tests & $<\mathbf{5}$ millions/ml & $>\mathbf{2 0}$ millions /ml \\
\hline Electroéjaculation & 11 & $1(9 \%)$ & $4(36 \%)$ \\
Physostigmine SC & 9 & $1(\mathbf{1 1 \%})$ & $2(22 \%)$ \\
Physostigmine IV & 6 & $3(50 \%)$ & $3(50 \%)$ \\
Midodrine IV & 26 & $4(\mathbf{1 5 \% )}$ & $\mathbf{1 4 ( 5 4 \% )}$ \\
Midodrine IV + Electroéja. & 13 & $2(15 \%)$ & $9(69 \%)$ \\
Midodrine IV + Vibro. & 3 & 0 & $3(100 \%)$ \\
\hline Total & $\mathbf{6 8}$ & $\mathbf{1 1 ( 1 6 \% )}$ & $\mathbf{3 5 ( 5 1 , 5 \% )}$ \\
\hline
\end{tabular}

Tableau 2 : Ejaculation antégrades et résultat du spermogramme suivant les méthodes utilisées.

\begin{tabular}{|c|c|c|c|c|}
\hline Méthodes & Nb de tests & Ej. antégrades & $\begin{array}{l}\text { Ej. anté., } \\
>20 \mathrm{M} / \mathrm{ml}\end{array}$ & $\begin{array}{c}\text { Ej. anté., }>20 \mathrm{MV} / \mathrm{ml}, \\
>20 \% \text { mobiles }\end{array}$ \\
\hline Electroéjaculation & 18 & $11(61 \%)$ & $4(22 \%)$ & $1(5,5 \%)$ \\
\hline Physostigmine SC & 24 & $9(37,5 \%)$ & $2(8 \%)$ & $2(8,5 \%)$ \\
\hline Physostigmine IV & 11 & $5(45,5 \%)$ & $2(18 \%)$ & $2(18 \%)$ \\
\hline Midodrine IV & 46 & $23(50 \%)$ & $11(24 \%)$ & $5(11 \%)$ \\
\hline Midodrine IV + Electroéja & 21 & $13(62 \%)$ & $9(43 \%)$ & $4(19 \%)$ \\
\hline Midodrine IV + Vibro. & 4 & $3(75 \%)$ & $3(75 \%)$ & $1(25 \%)$ \\
\hline Total & 124 & $64(52 \%)$ & $31(25 \%)$ & $15(12 \%)$ \\
\hline
\end{tabular}

c) La Midodrine (Guthron) : dans la série de JoNAS [16], l'usage de la Midodrine en une seule administration intra-veineuse a permis d'induire une éjaculation antégrade dans $58 \%$ des cas (7 sur 12). La Midodrine intraveineuse est surtout efficace lors de lésions de niveau T11 à L1 : 75\% d'éjaculations antégrades (12/16 tests) par rapport à $42 \%$ lors de lésions de niveau T1 à T10 (10/24 tests). Elle est peu efficace (5 échecs sur 6 tests) lors de lésions cervicales. Son association à l'électro-éjaculation améliore les résultats pour les lésions de niveau T1 à T10 (7 éjaculations antégrades sur 11 tests, soit $64 \%$ ). Dans notre série, cette technique a surtout été employée avec un délai supérieur à 6 mois par rapport à la lésion médullaire.

En ce qui concerne la numération des spermatozoïdes, nous avons noté une amélioration des taux d'éjaculations antégrades et rétrogrades avec plus de 20 millions de spermatozoïdes à la suite de l'association à l'électro-éjaculation. Les effectifs lors de l'association au vibro-massage sont trop petits pour pouvoir en tirer une conclusion.

La numération, qui ne témoigne que très partiellement de la qualité du sperme, n'est pas meilleure pour la Midodrine utilisée seule par rapport à l'électro-éjaculation, contrairement à l'association Midodrine électro-éjaculation. En ce qui concerne la mobilité, sous réserve de la valeur seuil retenue et de la difficulté à établir des normes, il existe une différence en faveur de la Midodrine et de l'association Midodrine électro-éjaculation.

Les éjaculations rétrogrades semblent moins fréquentes lors de l'association de la Midodrine à d'autres méthodes.

\section{LA PONCTION DES DEFERENTS}

L'équipe du Kremlin-Bicêtre a rapporté en 1990 le résultat de 10 ponctions chez des 
paraplégiques avec des lésions de niveaux T12-L1-L2. Dans 3 cas, il n'a pas été retrouvé de spermatozoïdes aux ponctions des deux déférents. A chaque ponction positive, il a été pratiqué une insémination. Une grossesse non menée à terme a été constatée. Il faut noter dans 4 cas sur 10 une amélioration après ponction de la qualité du sperme permettant la congélation. Cependant, comme le souligne Françors, les quantités restent modestes et les possibilités de nouvelles inséminations réduites.

Une grossesse multiple menée à terme (3 enfants) a été rapportée récemment par une équipe australienne, à la suite d'une ponction de sperme au niveau de la jonction épididymo-déférentielle chez un tétraplégique suivie d'un transfert intra-tubaire de gamètes.

\section{CONCLUSION}

La biopsie testiculaire nous a appris qu'il existait une lésion testiculaire d'origine neurologique, parfois irréversible, mais il n'a jamais été possible de définir à partir de quel stade il y avait irréversibilité.

Les lésions du testicule endocrine ont été réversibles sous stimulation ou spontanément.

Le diagnostic de stérilité masculine a eu des conséquences néfastes à un stade précoce de la paraplégie, parfois avec éclatement $d u$ couple. Aucun patient dont le pronostic de procréation était mauvais n'a voulu refaire de bilan de stérilité, ni se prêter aux essais d'éjaculation provoquée.

Il est donc bien préférable de rester très discret dans le pronostic de procréation, de se limiter au bilan endocrinien classique, testostérone, prolactine, FSH LH, la FSH étant l'élément essentiel, d'essayer à tout prix d'obtenir une éjaculation dans les conditions les plus simples possibles, en passant par l'amélioration de son érection si nécessaire par auto-injection intra-caverneuse, pour terminer par la biopsie de testicule réalisée en même temps que la ponction déférentielle si le bilan hormonal est correct et les essais ejjaculatoires infructueux.

Chez le paraplégique, comme chez tout homme infertile, la statistique n'a pas de valeur prédictive définitive. Elle doit guider les choix thérapeutiques. L'objectif reste l'obtention d'une grossesse. Il faut cependant toujours savoir à quel prix : financier, sociologique et surtout psychologique et avec quel risque. Il faut aussi savoir que le paraplégique doit respecter toute obligation de l'homme stérile : le traitement d'une prostatite, d'une escarre, mais aussi de sa dentition, l'arrêt du tabac peut faire diminuer des FSH élevés.

\section{REFERENCES}

1. BECKERMAN H., BECHER J., LANKHORST G.J. : The effectiveness of vibratory stimulation in anejaculatory men with spinal cord injury. Review article. In : Paraplegia, 1993, 31 : 689-699.

2. BERARD E., PERRIN B., LERICHE A., MINAIRE P., GIRARD R., BOURRET J. : Semiological value of painful sensitivity of the testis and spinal cord injuries. In : Paraplegia, 1978-1979, 15 : 373-378.

3. BERETTA G., CHELO E., ZANOLLO A. : Reproductive aspects in spinal cord injured males. In : Paraplegia, 1989, 27 : 113-118.

4. BORS E., ENGLE T., ROSENQUIST C., HOLLIGER V.H. : Fertility in paraplegic males. A preliminary report of endocrine studies. In : J. Clin. Endoc., 1950, $10: 381-398$.

5. BRINDLEY G.S. : The fertility of men with spinal cord injuries. In : Paraplegia, 1984, 22 : 337-348.

6. BRINDLEY G.S. : Reflex ejaculation under vibratory stimulation in paraplegic men. In : Paraplegia, 1981, 19, 5 : 299-302.

7. BRINDLEY G.S., SAUERWEIN D., HENDRY W.F. : Hypogastric plexus stimulators for obtaining semen from paraplegic men. In : Br. J. Urol., 1989, 64: 72-77.

8. CHAPELLE P.A. et al. : Neurological correlations of ejaculations and testicular size in men with complete spinal cord section. In : J. Neurol. Neurosur. Psychiat., 1988, 51 : 197-202.

9. CHAPELLE P.A., JONDET M., DURAND J., GROSSIAD A. : Pregnancy of the wife of a complete paraplegic by homologeous insemination after an intrathecal injection of neostigmine. In : Paraplegia, 1976, $14: 173-177$. 
10. COMARR E., A., BORS E. : Spermatocystography in patients with spinal cord injuries. In : J. Urol., $1955,73, \mathrm{n}^{\circ} 1: 172-178$.

11. DALAGE C., TANCHE M. : Effets de la destruction de la moelle épinière sur le conditionnement testiculaire du chien. In : J. Physiol. Paris, 1959, 51 : 441-442.

12. ENGH E., CLAUSEN O.P.F., PURVIS K., STIEN R. : Sperm quality assessed by flow cytometry and accessory sex gland function in spinal cord injured men after repeated vibration-induced ejaculation. In : Paraplegia, 1993, $31: 3-12$.

13. FRANCOIS $N$. et al : L'éjaculation par le vibromassage chez le paraplégique à propos de 50 cas avec 7 grossesses. In : Ann. Med. Phys., 1980, 23 : 24-36.

14. GUTTMANN L., WALSH J.J. : Prostigmine assessement test of fertility in spinal man. In : Paraplegia, 1971, $1: 39-50$.

15. HORNE H.W., PAUL D.P., MUNRO D. : Fertility studies with traumatic injuries of the spinal cord and cauda equina. In : New Eng. J. Med., 1948, 237 : 959-961.

16. JONAS D., LINZBACH P., WEBER W. : The use of midodrin in the treatment of ejaculation disorders following retroperitoneal lymphadenectomy. In : Eur. Urol., 1979, 5 : 184-187.

17. LERICHE A., BERARD E., VAUZELLE J.L., MINAIRE P., GIRARD R., ARCHIMBAUD J.P., BOURRET J. : Histological and hormonal testiculars changes in spinal cord patients. Congrès international de Paraplégie de Toronto 1976. In : Paraplegia, 1977-78, 15 : 274-279.

18. MORALES P., HARDIN J. : Scrotal and testicular temperature studies in paraplegia. In : J. Urol., 1958 , vol $79, \mathrm{n}^{\circ} 6$, june : $972-976$.

19. OHL D.A., BENNETT C.J., MC CABE M., MENGE A.C., MC GUIRE E.J. : Predictors of success in electroejaculation of spinal cord injured men. In : J. Urol., 1989, 142, 1483-1486.

20. OTANI ET AL. : A paraplegic fathering a child after an intrathecal injection of neostigmine : Case report. In : Paraplegia, 1985, $23: 32-37$.

21. SARKARATI M., ROSSIER A.B., FAM B.A. Experience in vibratory and electroejaculation techniques in spinal cord injury patients : a preliminary report. In : J. Urol., 1987, $138: 59-62$.

22. SIOSTEEN A., FORSSMAN L., STEEN Y., SULLIVAN L., WICKSTR OM : Quality of semen after repeated ejaculation treatment in spinal cord injured man. In : Paraplegia, 1990, 28 : 96-104.

23. SPIRA R. : Artificial insemination after intrathecal injection of neostigmine in a paraplegic. In : Lancet, 1956, $1: 670-671$.
24. THOMAS R.J.S., MC LEISH G., MC DONALD G. : Electroejaculation of the paraplegic male followed by pregnancy. In : Med. J. Australia, 1975, 2 : 798-799.

25. TSUJI I., KNAKAJIMA MORIMOTO : The sexual function in patients with spinal cord injuries. In : J. New Ment. Dis., 1960, $131: 121-123$.

\section{ABSTRACT \\ Male fertility and paraplegia}

Albert LeRICHE, Marie-Françoise MonNET, Bertrand Pogu, Geneviève PILONCherRY

\section{Hospices Civils de Lyon; Hôpital Henry Gabrielle, Unité d'Urologie, Route de Vourles, 69230 Saint Genis Laval}

In this article, the paraplegics' possibilities for procreation will be examined.

A first chapter will deal with the testicle functioning along with the study of hormonal measuring and testicle histology (study involving 100 patients). As a result, it appears that, after a testicle biopsy, there is a testicle lesion of neurological origin that is sometimes irreversible.

On the contrary, the endocrinal testicle lesions have proved reversible under stimulation or spontaneously.

A second chapter will deal with the different techniques to obtain an ejaculation and the semen analysis.

The techniques that were used being : massage by vibrator, electroejaculation, ejaculation through pharmacological stimulation. The study focuses on 124 ejaculatory tests among which 64 forward ejaculations (52\%) and 4 bachward ejaculations $(3 \%)$ were obtained. The resulting semen analysis revealed a spermatozoon count greater than 20 millions per millilitre (that is $51,5 \%$ ) and smaller than 5 millions in 11 cases $(16 \%)$.

Key-words : Paraplegia, male fertility, ejaculatory. 